\title{
Multilabel immunofluorescence and antigen reprobing on formalin-fixed paraffin-embedded sections: novel applications for precision pathology diagnosis
}

\author{
Jie Pan ${ }^{1,5}$, Cornelia Thoeni ${ }^{2,3,5,6}$, Aleixo Muise ${ }^{2,3,6}$, Herman Yeger $^{4}$ and Ernest Cutz ${ }^{1}$ \\ ${ }^{1}$ Division of Pathology, The Hospital for Sick Children, Toronto, ON, Canada; ${ }^{2}$ Division of Gastroenterology, \\ Hepatology and Nutrition, The Hospital for Sick Children, Toronto, ON, Canada; ${ }^{3}$ Program in Cell Biology, \\ The Hospital for Sick Children, Toronto, ON, Canada and ${ }^{4}$ Program in Developmental and Stem Cell Biology, \\ The Hospital for Sick Children, Toronto, ON, Canada
}

\begin{abstract}
We report new methods for multilabel immunofluorescence (MIF) and reprobing of antigen epitopes on the same formalin-fixed paraffin-embedded (FFPE) sections. The MIF method includes an antigen-retrieval step followed by multilabel immunostaining and examination by confocal microscopy. As examples, we illustrate epitopes localized to the apical and basolateral membranes, and the cytoplasm of enterocytes of normal small intestine and in cases of congenital enteropathies (microvillous inclusion disease and congenital tufting enteropathy). We also demonstrate localization of the bile salt excretion pump protein (BSEP) in bile canalicular membrane of normal hepatocytes and in cases of primary sclerosing cholangitis. To demonstrate colocalization of cytoplasmic and nuclear epitopes we analyzed normal control and hyperplastic pulmonary neuroendocrine cells (PNEC) and neuroepithelial bodies (NEBs), presumed airway sensors in the lungs of infants with bronchopulmonary dysplasia (BPD). As cytoplasmic markers we used anti-bombesin or anti-synaptic vesicle protein 2 (SV2) antibody, respectively, and for nuclear localization, antibodies against neurogenic genes mammalian achaete-scute homolog (Mash1) and prospero homeobox 1 (Prox1), essential for NEB cells differentiation and maturation, hypoxia-inducible factor $1 a($ HIF1a) a downstream modulator of hypoxia response and a proliferation marker Ki67. The reprobing method consisted of removal of the previously immunolabeled target and immunostaining with different antibodies, facilitating colocalization of enterocyte brush border epitopes as well as HIF1a, Mash1 and Prox1 in PNEC/NEB PNEC and NEBs. As these methods are suitable for routine FFPE pathology samples from various tissues, allowing visualization of multiple epitopes in the same cells/sections with superior contrast and resolution, they are suitable for a wide range of applications in diagnostic pathology and may be particularly well suited for precision medicine diagnostics.
\end{abstract}

Modern Pathology (2016) 29, 557-569; doi:10.1038/modpathol.2016.52; published online 4 March 2016

The last decade has witnessed great advances in immunohistochemical (IHC) methods, particularly, as applied to diagnostic pathology. The most commonly used procedure is indirect immunoperoxidase (IP) method with or without amplification

Correspondence: Dr E Cutz, MD, Division of Pathology, The Hospital for Sick Children, 555 University Ave., Toronto, ON M5G 1X8, Canada.

E-mail: ernest.cutz@sickkids.ca

${ }^{5}$ These authors contributed equally to this work.

${ }^{6}$ Participant in the InterNational Early Onset Pediatric IBD Cohort Study (www.NEOPICS.org).

Received 13 November 2015; revised 29 January 2016; accepted 29 January 2016; published online 4 March 2016 step that has greatly improved the sensitivity and specificity of epitope detection in a variety of cells and tissues. ${ }^{1}$ However, the use of the IP method for the detection of multiple epitopes in the same section is complex and with variable success, as it requires the use of different chromogens. On the other hand, the use of immunofluorescence (IF) methods in pathology diagnostics have been limited to frozen tissue sections, which are not always available. Previous experience with the use of the IF method on FFPE sections has been unsatisfactory, as such samples exhibit high background autofluorescence. However, an IF method for the use on FFPE sections was reported as a salvage technique for renal biopsies, in cases where frozen tissue was 
inadequate. ${ }^{2,3}$ This method uses a pronase digestion step to unmask immunoglobulin epitopes in the glomeruli and conventional fluorescence microscopy with the results comparable to routine frozen sections.

Traditionally, archival pathology samples are stored as FFPE tissues providing a wealth of valuable clinical material that is now being used with increasing frequency for molecular analysis. ${ }^{4} \mathrm{We}$ describe a multilabel immunofluorescence (MIF) method for confocal microscopy that offers several advantages over conventional IP immunoperoxidase or IF immunoflourescence methods including high resolution and improved contrast. Although MIF methods and confocal microscopy are being used extensively in research studies by cell and molecular biologists; thus far, these methods have not yet been widely adapted in diagnostic pathology. ${ }^{5}$ Here we report two new methods for MIF applicable to the investigation of routinely fixed and processed normal and pathological human tissue samples. The methods are relatively simple consisting of an antigen-retrieval step to unmask the epitope and to reduce background autofluorescence, followed by a
MIF procedure using antibodies to multiple epitopes. The subsequent examination of sections by confocal microscopy provides sharp contrast high-resolution images without background staining. In addition, the resulting digital images are suitable for morphometric analysis. ${ }^{5}$ The antigen-reprobing method, based on similar principles as used for western blot, allows localization of multiple epitopes in the same cellular structures. Reprobing of multiple targets in the exactly same cell/tissue location on the same section (instead of labeling multiple/serial tissue sections) offers several advantages, especially for samples from rare diseases or when samples are of small size and volume.

As examples of application of these methods we demonstrate localization of markers for apical and basolateral membrane as well as cytoplasmic epitopes in enterocytes of normal small intestine and in cases of congenital enteropathies including microvillous inclusion disease (MVID) and congenital tufting enteropathy (CTE). ${ }^{6,7} \mathrm{We}$ also demonstrate localization of the bile salt excretion pump (BSEP) in the bile canalicular membrane of normal hepatocytes

Table 1 Multiple target immnolabeling protocol for FFPE sections

Flow diagram for multilabel immunofluorescence and stripping, and reprobingof immunostainted FFPE sections

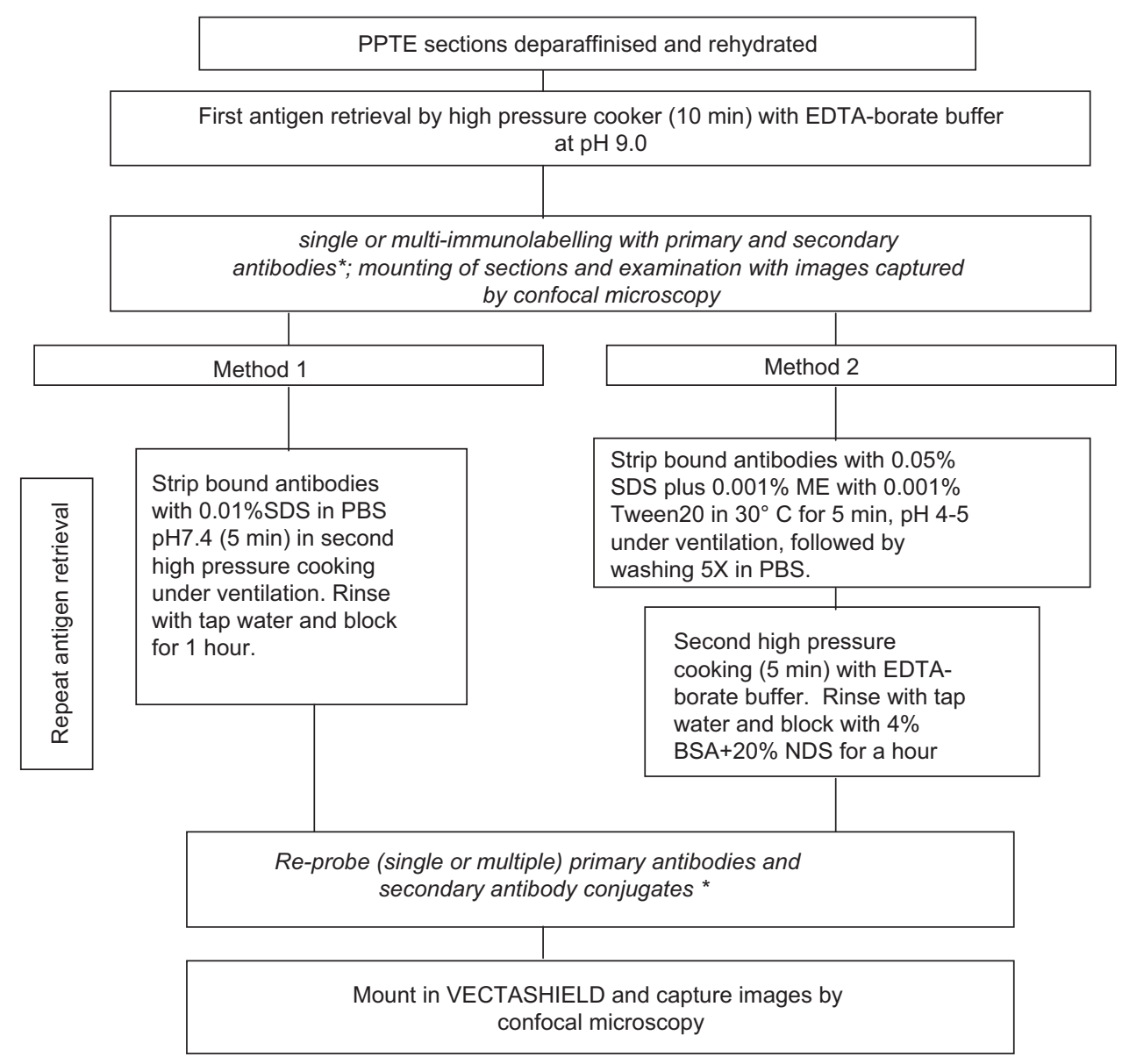


Table 2 Antibody sources

\begin{tabular}{lcl}
\hline & Dilutions & Sources \\
\hline Primary antibodies & & \\
Rb anti-EPCAM pAb & $1: 100$ & Sigma Aldrich \\
Ms anti- $\beta$-catenin mAb & $1: 100$ & BD Transduction \\
& & Labs \\
Ms anti-Villin mAb & $1: 100$ & Serotec \\
Ms anti-LAMP2 mAb & $1: 100$ & Abcam \\
Rb anti-Na/K ATPase pAb & $1: 100$ & Abcam \\
Rb anti-Myosin 1C pAb & $1: 100$ & Abcam \\
Rb anti-Syntaxin 3 mAb & $1: 100$ & Abcam \\
Rb anti-Bombesin pAb & $1: 100$ & Kamiya Biomed Co \\
Ms anti-Synaptic vesicle & $1: 100$ & Hybridoma Bank \\
protein 2 mAb (SV2) & & \\
Ms anti-Mash1 mAb & $1: 250$ & BD Pharmingen \\
Rb anti-Prox1 pAb & $1: 400$ & Abcam \\
Rb anti-Ki67 pAb & $1: 500$ & Dako \\
Rb anti-HIF1 $\alpha$ pAb & $1: 1000$ & Abcam \\
& & \\
Secondary antibodies & & \\
Anti-rabbit IgG ALEXA 568 & $1: 500$ & Life Technologies \\
Anti-mouse IgG ALEXA 488 & $1: 500$ & Life Technologies \\
Anti-mouse IgG H+L-biotin & $1: 200$ & Jackson Laboratories \\
Anti-rabbit IgG H+L-biotin & $1: 200$ & Jackson Laboratories \\
Anti-rabbit IgG H+L- & $1: 100$ & Jackson Laboratories \\
Rodamine & & \\
Anti-rabbit IgG H+L-FITC & $1: 200$ & Jackson Laboratories \\
Anti-rabbit IgG ALEXA 680 & $1: 200$ & Jackson Laboratories \\
Anti-mouse IgG H+L-Texas & $1: 1000$ & Jackson Laboratories \\
Red & & \\
Streptavidin-Texas Red X & $1: 1000$ & Invitrogen \\
RedDot-2 dye (697 nm) & $1: 1000$ & Biotium \\
\hline & & \\
\hline
\end{tabular}

and in cases of primary sclerosing cholangitis (PSC). In the lung, we illustrate expression of cytoplasmic neuroendocrine markers bombesin or anti-synaptic vesicle protein 2 (SV2) together with nuclear epitope localization of critical transcription factors including mammalian achaete-scute homolog (Mash1), prospero homeobox 1 (Prox1), proliferation marker Ki67 and hypoxia-inducible factor $1 \alpha$ (HIF1 $\alpha$ ) in controls and hyperplastic PNEC and NEBs in infants with bronchopulmonary dysplasia (BPD). ${ }^{8,9}$ Thus, these new methods are applicable to a wide range of normal and pathological tissue samples providing multilabel localization of different epitopes in various cell compartments that are enhanced by confocal imaging with superior contrast and resolution suitable for cellular and molecular studies including precision pathology diagnosis.

\section{Materials and methods}

Human tissue samples were retrieved from the files of the Division of Pathology, The Hospital for Sick Children. The samples of normal, control small intestine mucosa included duodenal biopsies from infants with GI symptoms but normal histology $(n=3)$ and for disease conditions, duodenal biopsies from infants with congenital enteropathies (MVID $(n=2)$ and CTE, $n=2)$ previously reported (ref. 10,11, and unpublished case). We analyzed duodenal biopsies from sibs with MVID with compound heterozygous mutations in the $M y o 5 B$ gene, as previously reported. ${ }^{10}$ The cases of CTE included one patient described previously, ${ }^{11}$ harboring a homozygous acceptor splice site mutation (c.427-1 $\mathrm{G}>\mathrm{A}$ ) in exon 4 of the EpCAM gene with loss of epithelial epithelial cell adhesion molecule (EpCAM) staining by IF. In the second, unpublished case of CTE no disease causing variant was found by whole-exome sequencing analysis as well as Sanger sequencing in genes previously reported to be mutated in congenital enteropathies. ${ }^{12}$ For histology analysis of liver biopsies, we used samples from cases showing normal liver histology $(n=3)$ and from cases of PSC $(n=2)$. For lung tissues, we used samples obtained at autopsy from infants $(n=3$, 5-10 month of age) who died of chronic lung disease of prematurity (BPD) and age-matched controls $(n=2)$ who died of non-pulmonary causes. All samples were fixed in $10 \%$ neutral buffered formalin, without methanol and embedded in paraffin using routine protocols. FFPE sections were cut at 5-7 $\mu \mathrm{m}$.

The use of human tissue samples was approved by the Research Ethics Board at the Hospital for Sick Children. For the use of small intestine biopsies from control and disease cases extensive and comprehensive consent was obtained. Informed consent to participate in research was obtained and a copy of the consent is available on the interNational Early Onset Pediatric IBD Cohort Study (NEOPICS) website at http://www.neopics.org/NEOPICS_Docu ments.html.

\section{MIF}

The details of MIF procedure are outlined in Table 1. As a first step, paraffin was removed using Xylene, and afterwards rehydrated with different percentages of ethanol. Antigen retrieval was performed with high-pressure cooking in EDTA-borax buffer (1 mM EDTA, $10 \mathrm{mM}$ borax (sodium tetraborate, Sigma, St Louis, MI, USA), $10 \mathrm{mM}$ boric acid (Sigma) with $0.001 \%$ Proclin 300 (Supleco, Bellefonte, PA, USA) at $\mathrm{pH}$ 9.0. To block non-specific staining, the slides were incubated for $1 \mathrm{~h}$ at room temperature in $5 \%$ BSA in $1 \times$ phosphate-buffered saline (PBS, Multi Cell) without $\mathrm{Ca}^{2+}$ and $\mathrm{Mg}^{2+}$ containing $15 \%$ goat serum. Primary antibody incubation was performed overnight at $4{ }^{\circ} \mathrm{C}$ (Table 2). On the following day, stained slides were washed three times for $10 \mathrm{~min}$ with $1 \times$ PBS without $\mathrm{Ca}^{2+}$ and $\mathrm{Mg}^{2+}$. Secondary antibody incubation was performed at room temperature in darkness for $1 \mathrm{~h}$, and slides were washed afterwards three times for $10 \mathrm{~min}$ in darkness. As a nuclear counterstain Hoechst 33342 Fluorescence Stain (Thermo Scientific) was used at a dilution of 1:15.000. Finally, sections were mounted overnight with Vector shield fluorescence mounting medium (Vector Labs). 
Table 3 Multiple target immnolabeling protocol for FFPE sections

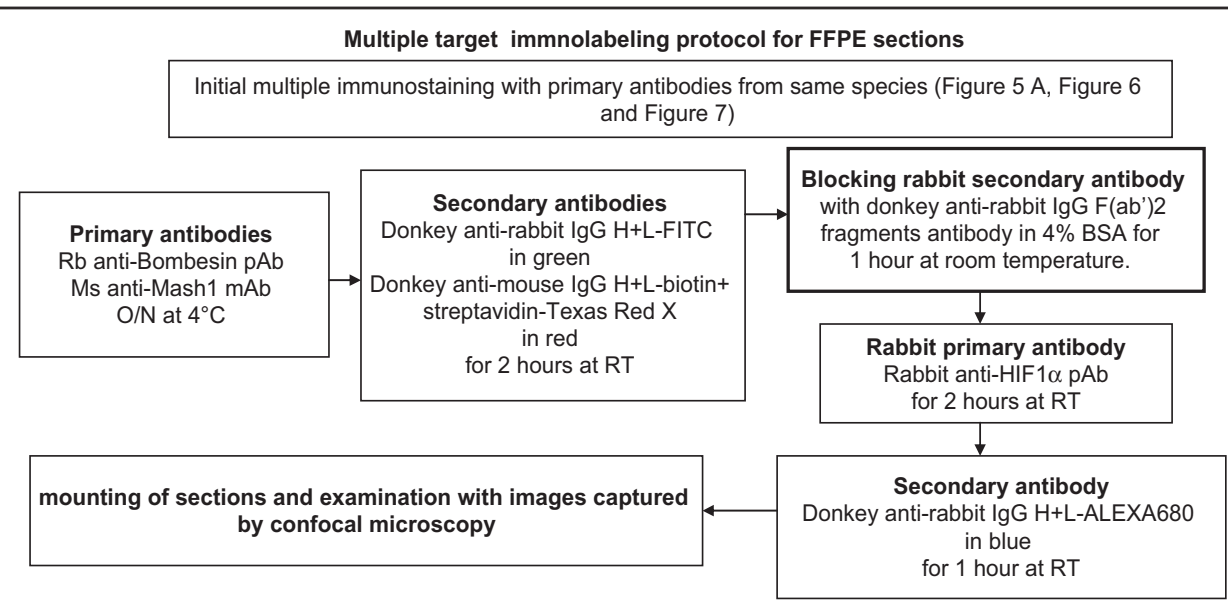

Re-probing after stripping initial antibodies (Figure 5B, Figure 6 and Figure 7)

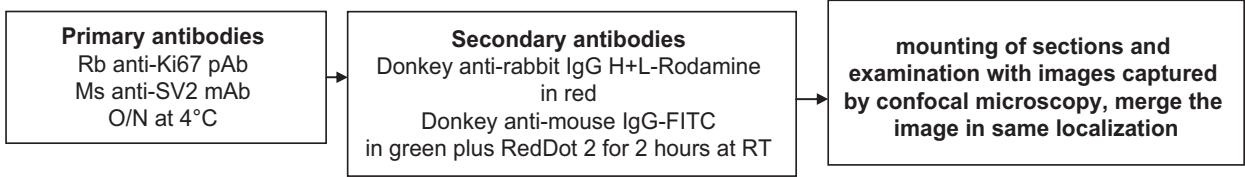

Stripping and Reprobing of Previously Immunostained Formalin-Fixed Paraffin-Embedded Tissue Sections

The general outline of the procedures used for the removal/stripping of previously immunolabeled qtargets is shown in Table 1 . To remove the coverslips, the slides were dipped in $100 \%$ xylene for a few seconds, and then the coverslip gently removed. For duodenal biopsy samples we used method 1, consisting of incubation in $0.01 \%$ sodium dodecyl sulfate (SDS, Sigma Aldrich) diluted in $1 \times$ PBS as a stripping solution and exposed sections for $5 \mathrm{~min}$ at $110^{\circ} \mathrm{C}$ in a high-pressure Dekloak chamber (Thermo Scientific) within a ventilation hood (Table 1). Afterwards the slides were washed for $5 \mathrm{~min}$ in cold tap water and blocked for $1 \mathrm{~h}$ in $5 \%$ BSA in $1 \times$ PBS containing $15 \%$ goat serum. Subsequently, the sections were incubated overnight with the first antibody at $4^{\circ} \mathrm{C}$ and further processed as described above.

For samples of lung, the procedure was slightly modified, using method 2 (Table 1). After three washes ( $5 \mathrm{~min} / \mathrm{each})$ with $\mathrm{PBS}$, the pre-warmed tissue striping solution $(0.05 \%$ SDS; $0.001 \%$ b-mercaptoethanol; 0.001 Tween20 in MiniQ water pH4.0 at $30^{\circ} \mathrm{C}$ ) was added on each slide and incubated for $5 \mathrm{~min}$ at room temperature under a V-hood. After washing the slides five times with PBS ( $5 \mathrm{~min} / \mathrm{each}$ ), we performed acid $\mathrm{pH}$ antigen retrieval in a high-pressure cooker as described above. After washing and blocking, the slides were ready for reprobing with different antibodies (Table 3).

\section{Confocal Microscopy}

Photomicrographs of immunostained sections of small intestine and liver were taken with an Olympus IX81 inverted fluorescence microscope equipped with a Hamamatsu C9100-13 back-thinned EM-CCD camera and Yokogawa CSU X1 spinning disk confocal scan head. Images were adjusted for contrast and brightness using the Volocity 6.1.1 version software (Perkin Elmer).

Fluorescent confocal images of PNEC/NEB cells immunostained for bombesin, SV2, Mash1, Prox1, HIF1 $\alpha$, or Ki67 in double/triple-immunostained sections were obtained using a Leica confocal laser scanning microscope (model TCS-SPE) and LAS-AF software, as previously reported. ${ }^{13}$ The variable excitation wavelengths of the krypton/argon laser were $488 \mathrm{~nm}$ for FITC conjugate, $568 \mathrm{~nm}$ for Texas Red complex and $695 \mathrm{~nm}$ for Alexa Fluor 680 conjugate/RedDot-2 (nuclear counterstaining).

\section{Results}

\section{MIF on Formalin-Fixed Paraffin-Embedded Tissue Sections}

Duodenal biopsy samples from normal controls stained with Hematoxylin and Eosin showed excellent cell and tissue preservation (Figure 1a). For demonstration of apical polarity of villous enterocytes, the samples were immunostained for villin, a marker of intestinal brush border and syntaxin3, a 

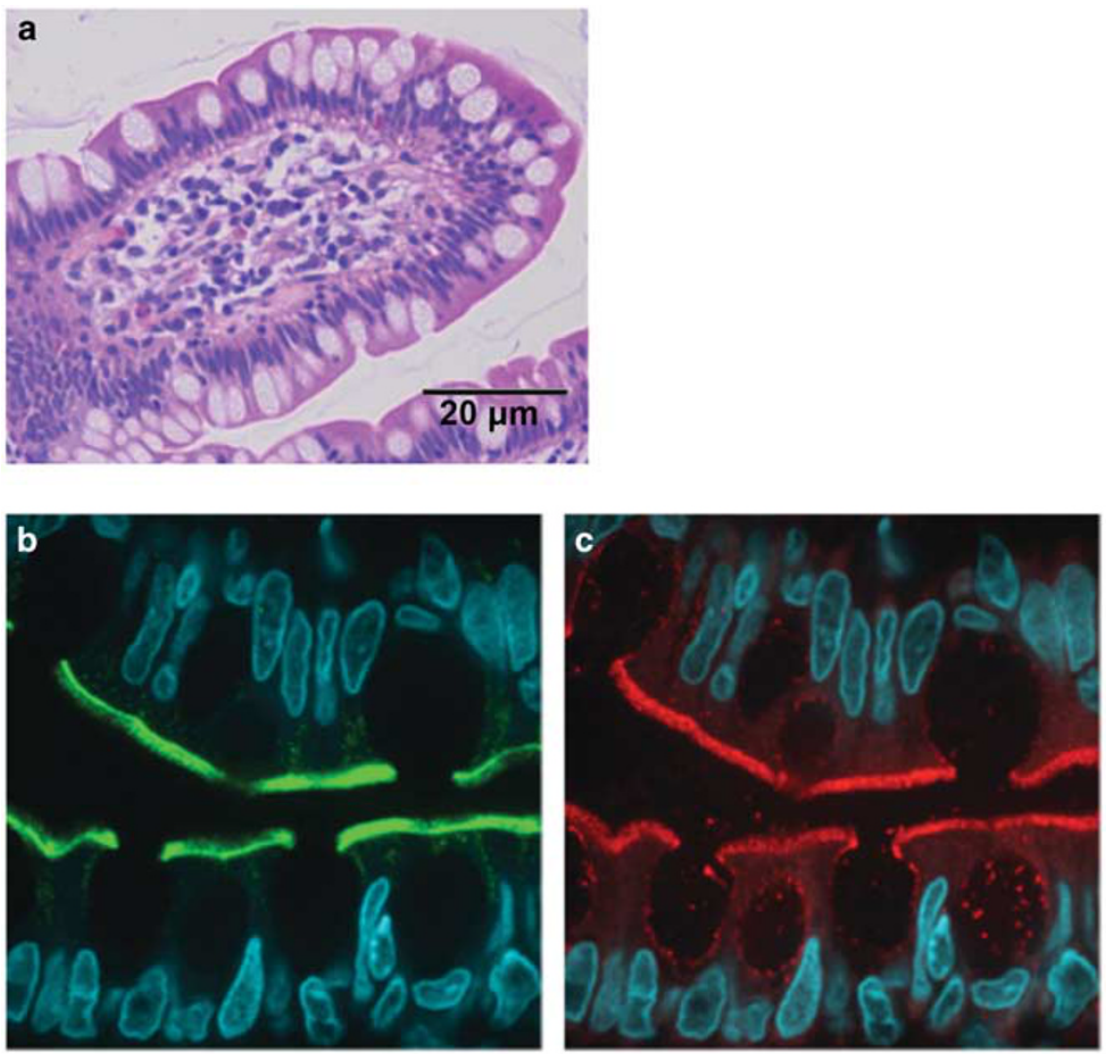

villin/syntaxin $3 /$ nuclei
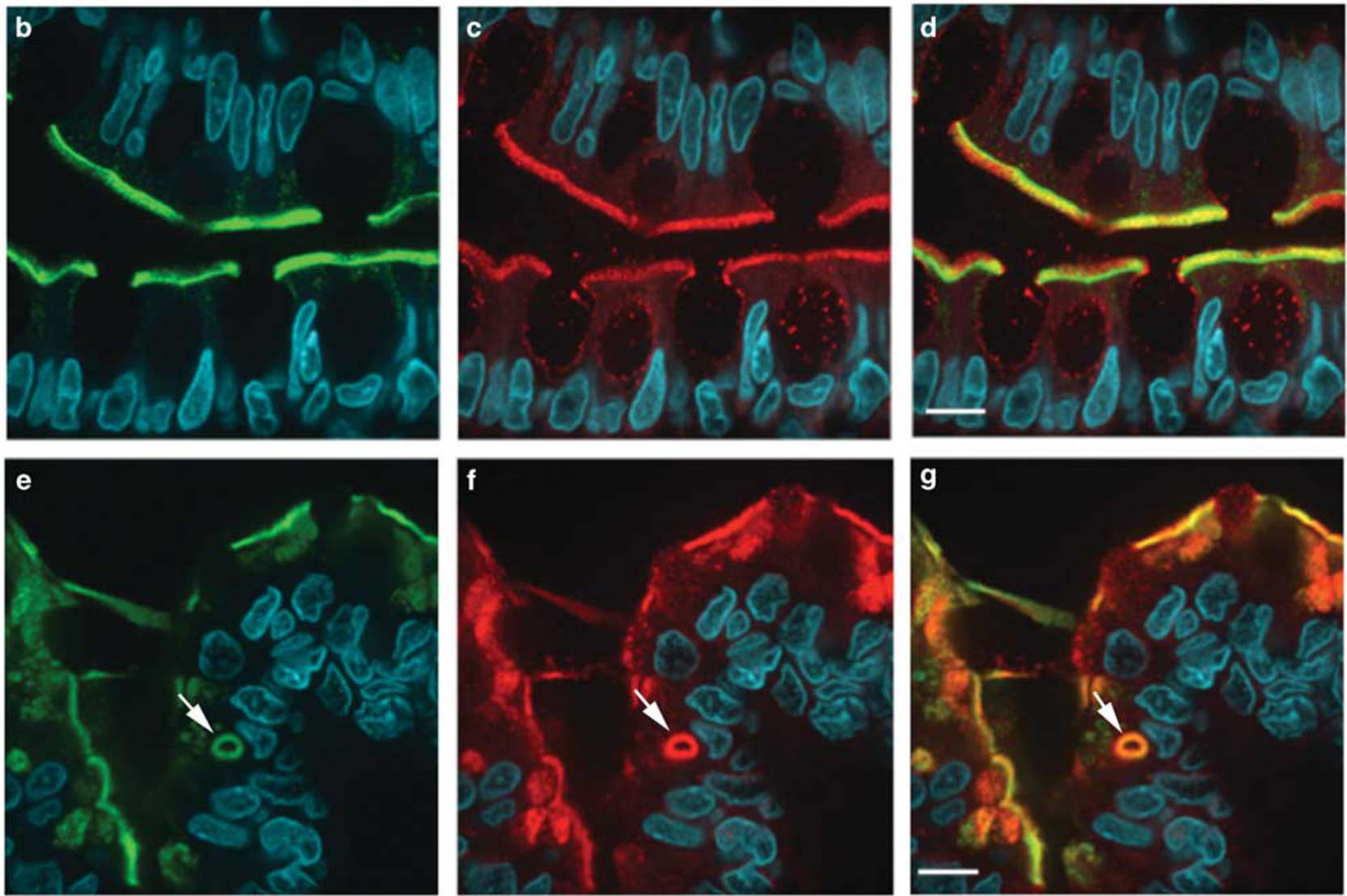

Figure 1 (a) Duodenal biopsy from normal control stained by Hematoxylin and Eosin showing part of a villus lined by enterocytes and goblet cells. Scale bar $=20 \mu \mathrm{m}$. (b-d) Multilabel immunofluorescence staining of control duodenal biopsy. (b) Immunostaining for villin localized apically in villus enterocytes marking brush border membrane; (c) Immunostaining for syntaxin3 a marker for exocytosis and recycling vesicles is colocalized with villin to brush border and subapical cytoplasm demonstrating the fusion of vesicles with the apical membrane. (d) Merged image demonstrating co-expression of villin and syntaxin3 in brush border membrane. (e-g) Multilabel immunofluorescence of duodenum biopsy from case of MVID. (e) Discontinuous and patchy immunostaining for villin in apical membrane of enterocytes and in microvillous inclusions (arrow). (f) Immunostaining for syntaxin3 shows similar pattern to villin including immunostaining of microvillous inclusions (arrow). (g) Merged image confirming colocalization pattern of villin and syntaxin3 in brush border membrane and microvillous inclusion (arrow). Villin immunostaining in green (ALEXA 488), syntaxin3 in red (ALEXA 568), and nuclei in blue (Hoechst). Scale bars $=10 \mu \mathrm{m}$.

marker for recycling and exocytotic vesicles (Figure 1c). In normal control samples, villus and crypt enterocytes (not shown), villin was localized to the apical brush border membrane (Figure 1b), whereas syntaxin3 was expressed both in brush border membrane as well as in vesicle structures in the subapical cytoplasm ((Figure 1c). As shown in merged image, syntaxin3 colocalized with villin, demonstrating the fusion of vesicles with the apical brush border membrane (Figure 1d). In cases of MVID, there was patchy expression of villin in enterocyte apical cytoplasm and the presence of occasional round, dough nut-like cytoplasmic microvillous inclusions (MI), a marker of this disease 

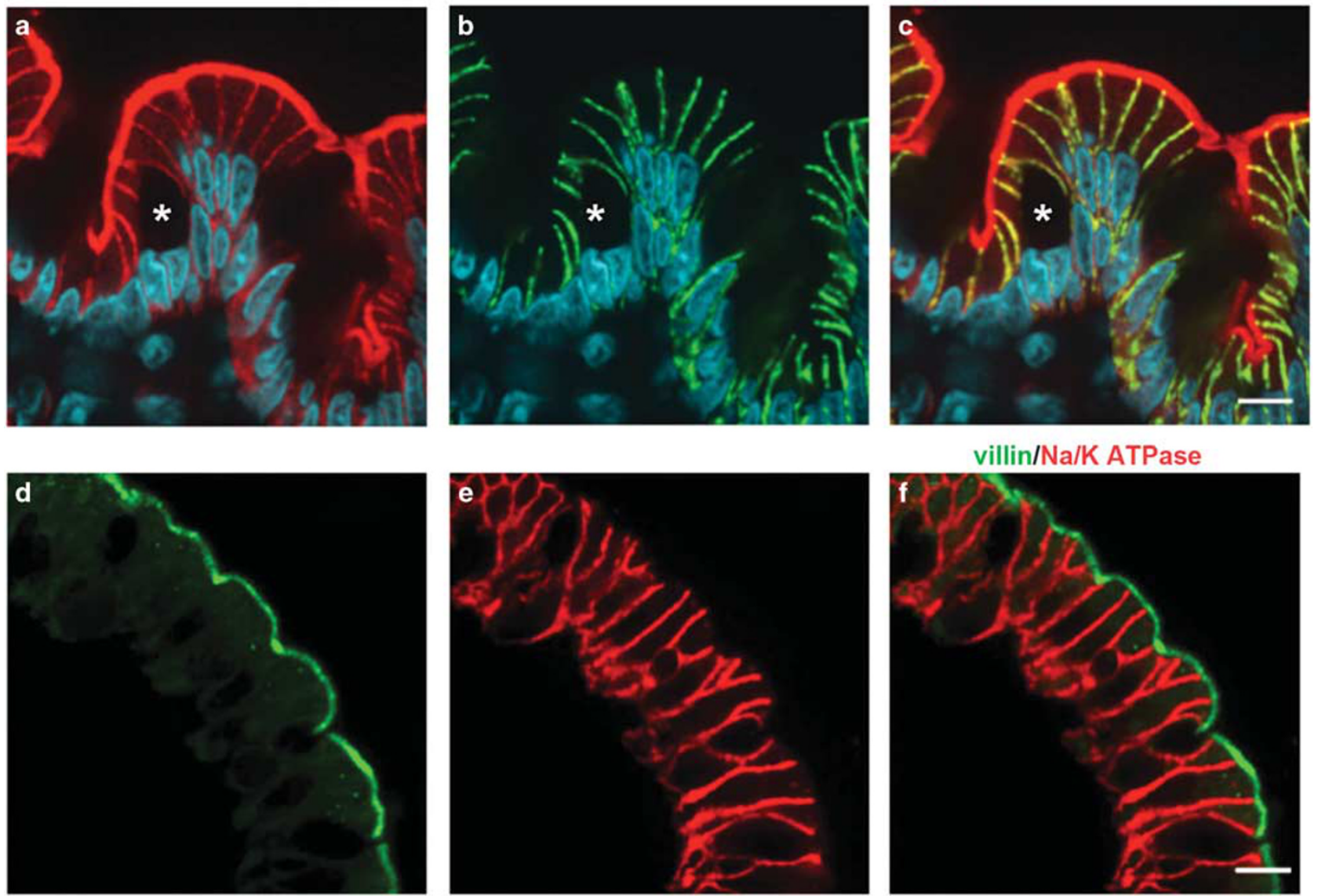

Figure 2 (a-f) Multilabel immunofluorescence of control duodenal biopsy. (a) Immunostaining for myosin1c, localized apically and basolaterally. (b) Immunostaining for beta-catenin, marker for cell-cell adhesion localized to basolateral membrane of enterocytes; (c) Merged image demonstrating colocalization of beta-catenin and myosin1c in basolateral membrane; (a-c) unstained patches of epithelium (asterisk) represent goblet cells. (d) The same section as in a, after stripping and immunostaining for villin demonstrating intact apical membrane epitope similar to Figure 1b. (e) Immunostaining of stripped section same as in A for Na/K ATPase shows strong expression and localization to basolateral membrane of enterocytes; (f) merged image with distinctive apical villin immunostaining and basolateral membrane localization of Na/K ATPase. Villin immunostaining in green (ALEXA 488) and Na/K ATPase in red (ALEXA 568). Scale bars $=10 \mu \mathrm{m}$.

(Figure 1e). Syntaxin 3 expression was similar to villin, in both apical surface and in MI (Figure $1 \mathrm{f}$ and g). The non-muscle motor protein, myosin1c that transports vesicles from the basolateral to the apical compartment showed localization to both apical and basolateral membranes of enterocytes (Figure 2a). On the other hand, immunostaining for cell adhesion molecule, beta-catenin was exclusively localized to the basolateral membrane (Figure 2b), where it colocalized with myosin1c (Figure 2c).

To demonstrate the versatility of our antigenreprobing method, the same section used for immunolocalization of epitopes shown in Figure 2a-c was stripped and reprobed with antibodies against villin that was localized to the brush border membrane and $\mathrm{Na} / \mathrm{K}$ ATPase exclusively localized to the basolateral membrane (Figure 2d, e and f). To assess visualization of cytoplasmic cell organelles, sections were immunostained for late endosomes/lysosomes (Lamp2), that were localized subapically in duodenal villus enterocytes (Figure 3a). Furthermore, immunostaining for EpCAM, involved in formation of tight junctions, ${ }^{14}$ was localized to the basolateral membrane of villus enterocytes (Figure $3 \mathrm{~b}$ and $\mathrm{c}$ ). Interestingly, in a case of CTE immunostaining for EpCAM was absent from the basolateral membrane of enterocytes, as previously reported, ${ }^{7}$ but in addition was mislocalized to subapical cytoplasmic inclusions that colocalized with Lamp2-positive aggregates representing lysosome-like structures (Figure 3d, e and f). Taken together, the findings in this patient with a new variant of CTE suggest disruption of cell polarity as shown by mislocalization of EpCAM protein in Lamp2 organelles and defects within the apical cell compartment.

To test the usefulness of our MIF method in localization of other plasma membrane proteins in different cells/tissues we assessed the expression of BSEP in the bile canalicular membrane of 

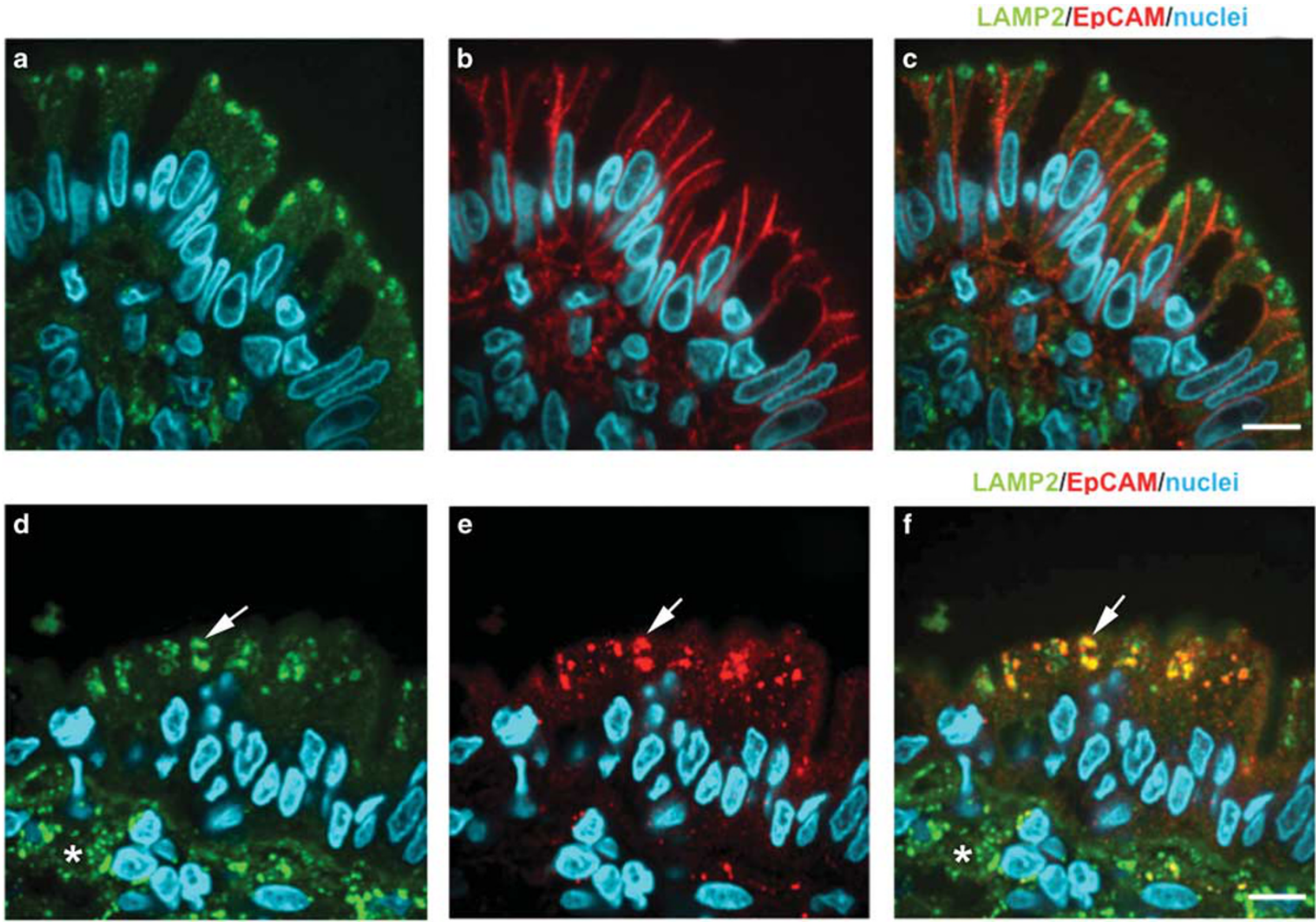

Figure 3 (a-c) Multilabel immunofluorescence stains of normal control duodenum for Lamp2 (a marker for late endosomes/lysosomes) localized subapically in villus enterocytes; (b) EpCAM (marker for epithelial cell adhesion molecule labeling tight junctions) localized to basolateral membrane of villus enterocytes. (c) Merged image with distinct separation of subapical cytoplasmic localization of Lamp2 and EpCAM immunostaining of basolateral membranes. (d-f) Immunolabeling for Lamp2 and EpCAM in duodenal biopsy from a patient with CTE. (d) Aggregates of Lamp2-positive lysosome-like inclusions in apical cytoplasm of villous enterocytes (arrow). Inflammatory cells in submucosa show Lamp2 positivity in cytoplasm (asterisk). (e) Immunostaining for EpCAM shows lack of immunostaining of basolateral membrane (compared with b) and EpCAM-positive aggregates are present in apical cytoplasm(arrow); (f) Merged image confirms colocalization of EpCAM in Lamp2-positive lysosomes (arrow). Lamp2 staining in green (ALEXA 488), EpCAM in red (ALEXA 568), and nuclei in blue (Hoechst). Scale bars $=10 \mu \mathrm{m}$.

hepatocytes. It should be noted that immunostaining for BESP is a useful marker in the diagnosis of congenital cholestasis syndromes. ${ }^{15}$ In sections of liver with normal histology shown in HE stains (Figure 4a), as expected, BSEP was localized to the bile canalicular membrane with sharp contrast and clear definition (Figures $4 \mathrm{~b}$ and $3 \mathrm{~d}$ reconstruction in Figure 4c). In contrast, in a case of PSC, HE hematoxilin and eosin staining showed cholestasis and immunostaning for BESEP protein revealed discontinuous, focally beaded staining pattern (Figure $4 \mathrm{~d}$ and e, and Figure $3 \mathrm{~d}$ reconstruction in Figure 4f).

In agreement with previous studies, PNEC/NEB in lungs of control infants and those with BPD showed strong cytoplasmic immunoreactivity for bombesin, the principal peptide produced by these cells in human lung, used here as a cytoplasmic marker for PNEC/NEB cells (Figure 5a). In cases of BPD, PNEC/ NEB cells showed striking hyperplasia compared with age-matched controls as previously reported. ${ }^{8,9}$ In lung sections from cases of BPD with double immunolabeling for bombesin and Mash1, the majority of PNEC/NEB cells were immunoreactive for both epitopes, suggestive of differentiation from precursor cells (Figure 5a). To identify proliferating cell populations in the same section as in Figure 5a, the section was stripped and reprobed with antibodies against SV2 (general neural and neuroendocrine cell marker) and an antibody against Ki67, expressed in the nuclei of proliferating cells. As expected, SV2 was localized in the cytoplasm of PNEC/NEB cells and also immunostained adjacent sub epithelial nerve fibers (Figure $5 b$ ). The proliferation marker Ki 67 was expressed in the majority of non-endocrine airway epithelial cells with only occasional PNEC co-expressing both Mash1 and Ki67 or Ki67 alone (Figure 5b). Generally, although in lungs of control infants most NEBs were usually made up of between 5 and 15 cells, in the infants 

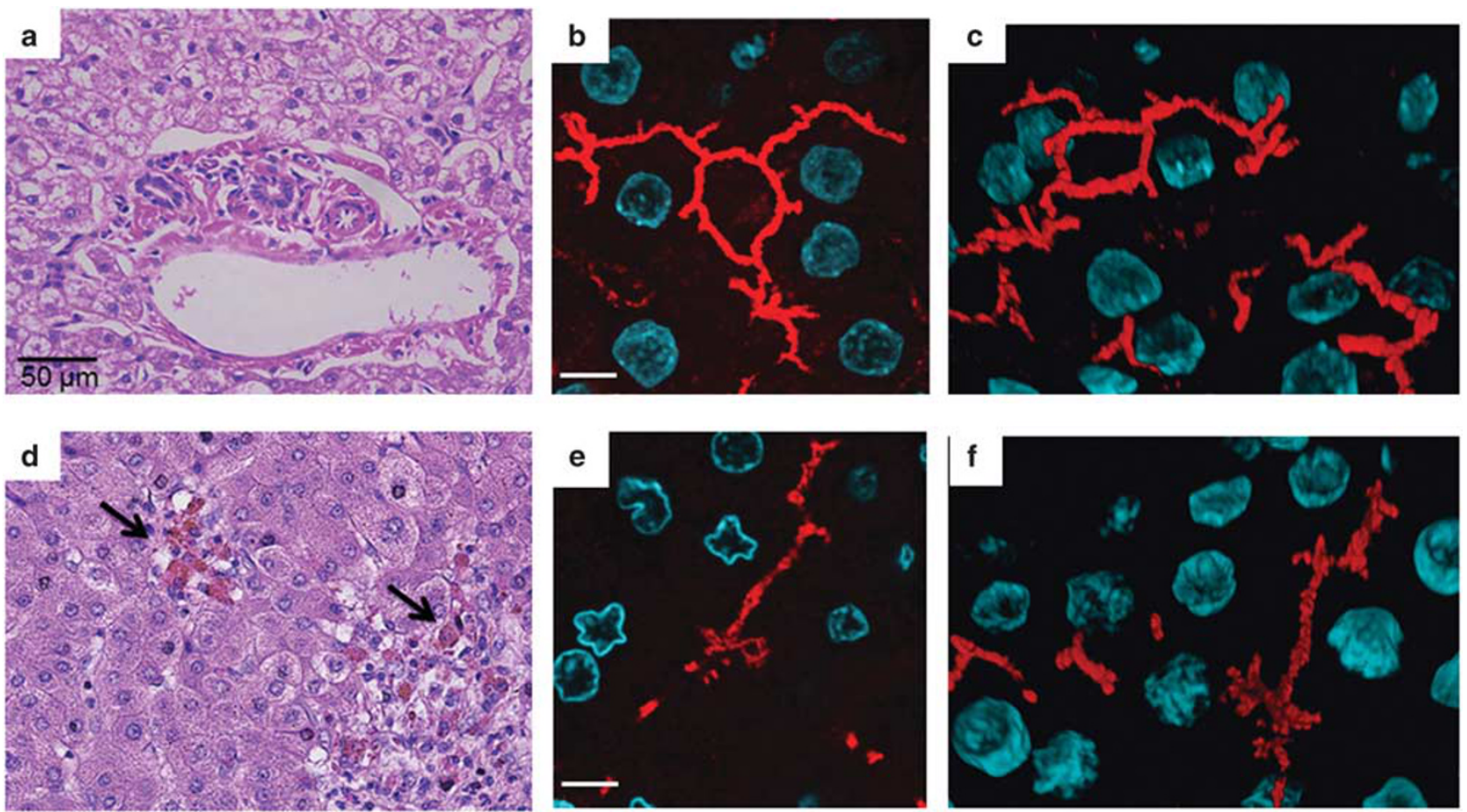

Figure 4 (a-c) FFPE section from control liver biopsy. (a) Hematoxylin and Eosin (HE) staining of liver biopsy shows well-preserved hepatocytes and portal structures. (b) Immunofluorescence staining for BSEP shows localization to bile canaliculi of hepatocytes forming a continuous network. (c) 3D reconstruction of control hepatocytes illustrating a normal bile canalicular system. (d-f) FFPE liver biopsy from a patient with PSC. (d) Hematoxylin and Eosin (HE) staining showing intrahepatic cholestasis (arrows). (e) Immunostaining for BSEP shows discontinuous staining of bile canaliculi. (f) 3D reconstruction of PSC hepatocytes illustrating a disrupted bile canalicular system. BSEP staining in red (ALEXA 568) and nuclei in blue (Hoechst). Confocal microscopy planes were merged as well as 3D reconstruction performed to enhance visualization of bile canaliculi in hepatocytes. Scale bars $=10 \mu \mathrm{m}$.

with BPD, majority of NEBs neuroepithelial bodies were hyperplastic and contained up to 50 cells (Figures 6 and 7). In agreement with previous studies, in control neonatal lungs only occasional NEB cells expressed Mash1 ( $<5 \%)$ (Figure 6a), whereas up to $50 \%$ expressed NEB cell maturation marker Prox1 (Figure 6b). ${ }^{16,17}$ In NEB cells of control lungs, expression of HIF1 $\alpha$ was identified in occasional cells where it was colocalized in Mash1- and Prox1-negative nuclei (Figure 6c). The merged image of triple immunostaning confirmed that each epitope (Mash1, Prox1, HIF1 $\alpha$ ) was localized in different NEB cells without apparent co-expression, possibly indicating fully mature differentiated state (Figure 6d).

In cases of BPD, more than half of NEB cells were immunoreactive for Mash1 with fewer Prox1-positive nuclei indicating increased recruitment from precursor cells (Figure 7a and b). The lower expression level of Prox1 is in keeping with its activity downstream of Mash1 and its involvement in NEB cell maturation. ${ }^{16}$ In contrast to controls, NEBs neuroepithelial bodies in BPD cases showed high expression levels of HIF $1 \alpha$ that closely correlated with Mash1 expression suggesting a hypoxia driven response (Figure 7c). However, occasional NEB cell nuclei showed either HIF1 $\alpha$ or Mash1 immunoreactivity only, likely reflecting their state of differentiation (Figure $7 \mathrm{a}$ and c). Co-expression analysis of Mash1, Prox1 and HIF1 $\alpha$ showed distinctive patterns with the majority of NEB cell nuclei $(\sim 90 \%)$ co-expressing Mash1 and HIF1 $\alpha$, with a few nuclei exhibiting either Mash1, HIF $1 \alpha$ or Prox1 expression alone (Figure $7 \mathrm{~d}$ ). It should be noted that neuroendocrine /neurogenic nuclear epitopes were exclusively expressed in PNEC/NEB cells, whereas in all other lung epithelial and mesenchymal cells, in both control and BPD lungs, no expression of these epitopes was seen.

\section{Discussion}

We describe two new IHC methods suitable for examination of routinely formalin-fixed and paraffin-embedded tissue sections from normal individuals and from patients with various disease conditions. Our MIF method is superior to conventional IF immunoflourescence on frozen tissues as formalin fixation provides excellent cytological preservation of cell structures and paraffin embedding facilitates tissue sectioning. The use of confocal imaging not only allows multiple immunolabelling on the same sections, but also, owing to a larger wave length spectrum for fluorescence excitation provides 

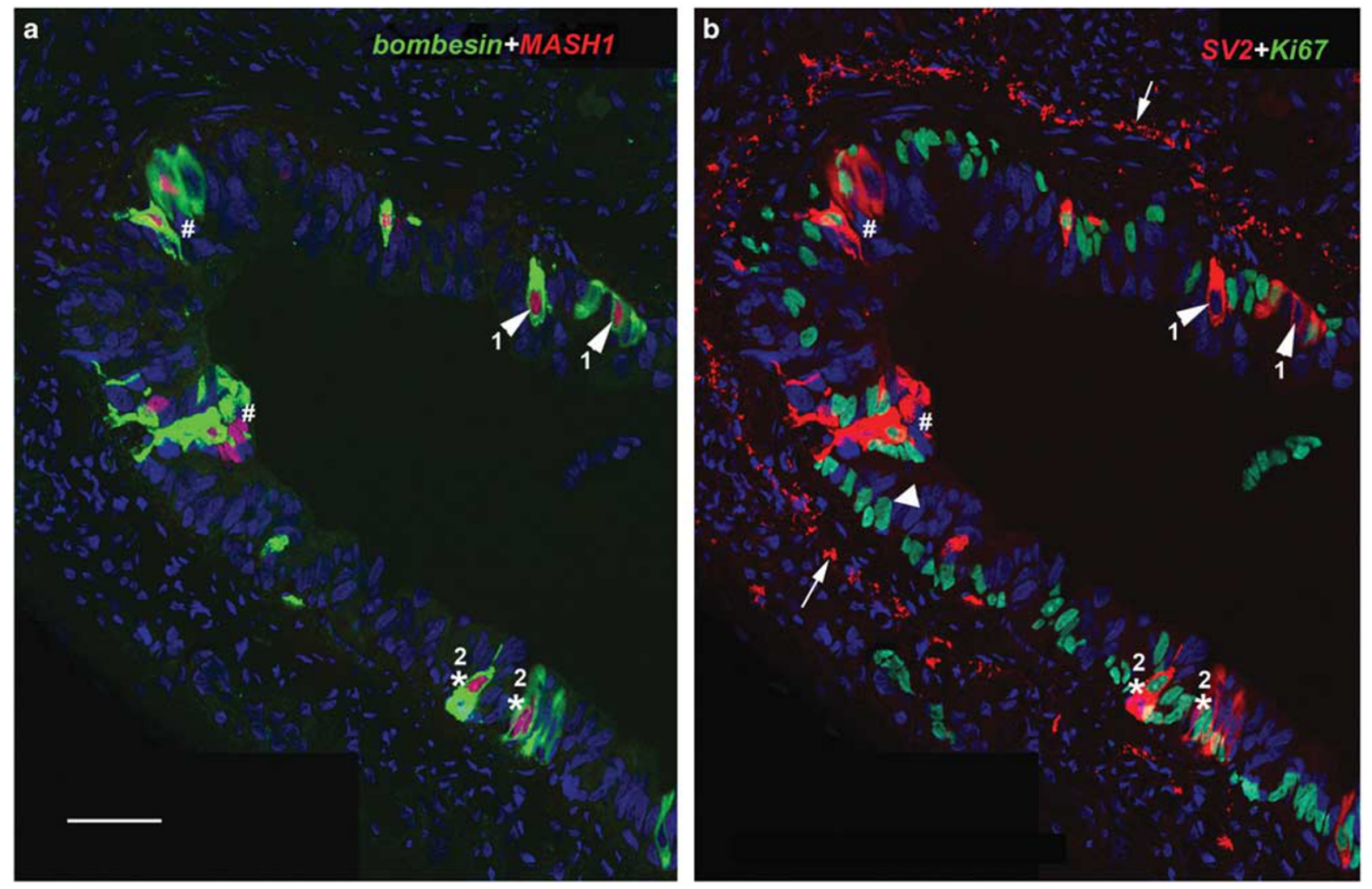

Figure 5 (a) Mulilabel immunofluorescence of lung section from 3-month-old infant with BPD immunostained for bombesin and Mash1. Airway epithelium containing bombesin immunoreactive solitary PNEC (arrow) and NEBs(\#) showing nuclear expression of Mash1; (b) same section as in a stripped and reprobed with antibodies against SV2 and Ki67. SV2 immunoreactivity is localized to bombesin immunoreactive PNEC/NEBs shown in Figure a. In addition, SV2 immunoreactive nerve fibers are present in peribronchial tissue (arrows). Majority of airway epithelial cells express Ki67, indicating active proliferation (open triangle). PNEC immunoreactive for both bombesin and SV2 (arrow with \#1) are positive for Mash1 (Figure a) but negative for Ki67 (Figure b). Different PNEC (asterisk \#2) co express both Mash1 and Ki67. Scale bar $=20 \mu \mathrm{m}$.

images with high resolution and contrast. Using this method, we show exquisite immunolocalization of various membranes, cytoplasmic and nuclear epitopes in the same cells from the same sections.

In samples of duodenal biopsies from normal controls we demonstrate immunolocalization of villin and syntaxin3 to the apical brush border, myosin1c to the apical brush border as well as basolateral membrane of villous enterocytes. In cases of MVID there was discontinuous villin and syntaxin3 immunostaining of apical membrane of enterocytes, consistent with focal loss of brush border and the presence of characteristic intracytoplasmic inclusions. These inclusions are usually sparsely distributed, affecting only $\sim 10 \%$ of villous enterocytes and hence are difficult to identify by routine IHC methods. Recent studies identified mutations in the $M Y O 5 B$ gene as a cause of most cases of MVID. 6,10,12 The loss of the MYO5B gene expression leads to defective cellular traffic and disrupted cell polarity of enterocytes. ${ }^{6,10,18}$ Mutation in the syntaxin 3 gene has been described recently in a case of variant form of MVID, presenting with milder clinical symptoms. ${ }^{19}$ We also show expression of cell adhesion molecules beta-catenin and EpCAM as well as an ion pump $\mathrm{Na} / \mathrm{K}$ ATPase to the basolateral membrane of normal enterocytes. In our case of a new variant of CTE, as expected, immunostaining for EpCAM was absent from the basolateral membrane, ${ }^{7}$ but in addition it was mislocalized to the cytoplasm within Lamp2 immunopositive aggregates representing early lysozymes. Based on these findings, we suggest that our MIF method is well suited for diagnostic work-up of congenital enteropathies where the sample size is often limited and frozen tissue in most cases is not available. Furthermore, studies can be performed on archival material in cases suspected of congenital enteropathy. ${ }^{12}$ We also demonstrate that the BSEP protein, physiologically located at the apical membrane of hepatocytes and a marker for intrahepatic bile canaliculi, ${ }^{15}$ can be assessed in FFPE liver biopsy samples to visualize the structure of bile canaliculi. This is of clinical relevance for patients with progressive familial intrahepatic cholestasis type 1 , a rare autosomal recessive liver disease 

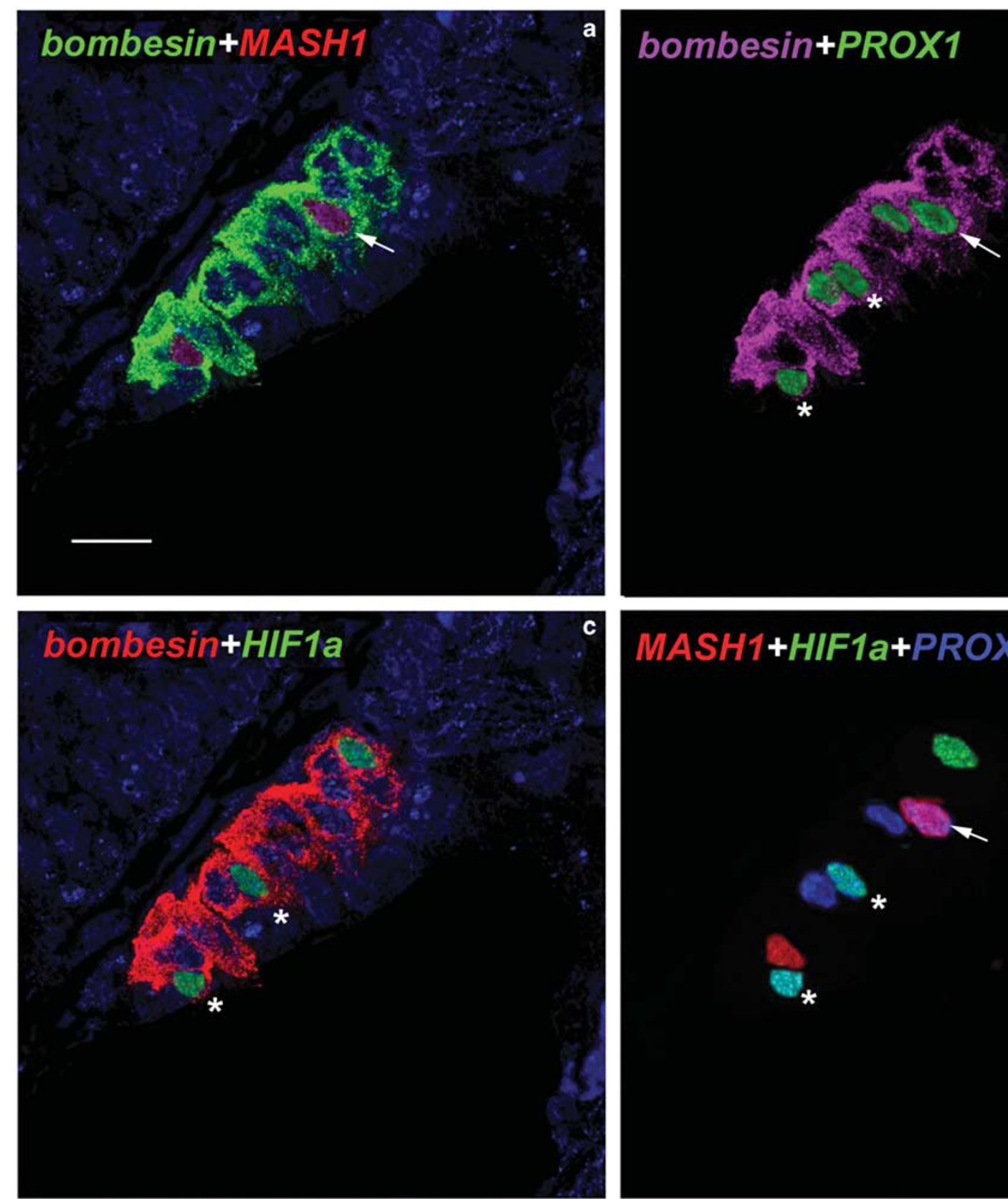

Figure 6 Tripple immunostaining of NEB containing up to 13 nuclei in lung of control 2-month-old infant. The section was first immunostained for bombesin (FITC, green), Mash1 (rhodamine, red), and HIF1 $\alpha$ (ALEXA 680, pseudogreen); after stripping the section was re probed with antibodies against bombesin (rhodamine, red) and Prox1 (FITC green). (a) Immunostaining for bombesin and Mash1 with two NEB nuclei-expressing Mash1; (b) Same section as in a, after stripping and reprobing using antibodies against bombesin and Prox1. Up to five NEB nuclei immunoreactive for Prox1, with only one expressing both Mash1 and Prox1 (arrow); (c) Section in a and b after stripping and reprobing for bombesin and HIF1 $\alpha$. Only three NEB nuclei are positive for HIF1 $\alpha$, with two co-expressing Prox1 and HIF1 $\alpha$ (asterisk), without co-expression of Mash1 and HIF1 $\alpha$. Scale bar $=10 \mu \mathrm{m}$.

characterized by progressive cholestasis owing to mutations in the BSEP gene, ${ }^{15}$ but also for other liver diseases where the intrahepatic bile canaliculi may be disrupted, as shown here in cases of PSC primary sclerosing cholingitis.

As examples of colocalization of different cytoplasmic and nuclear epitopes in the same sections/cells we have investigated PNEC/NEBs in control human lungs and in cases of BPD. Although the functional relationship between solitary PNEC and NEB cell clusters is presently unclear, NEBs neuroepithelial bodies are presumed polymodal airway sensors responding to a variety of stimuli including hypoxia, hypercarbia, acidosis, and mechanical stretch. ${ }^{9,20}$ PNEC/NEB produce amine (serotonin) and peptides, particularly bombesin in human lung and 

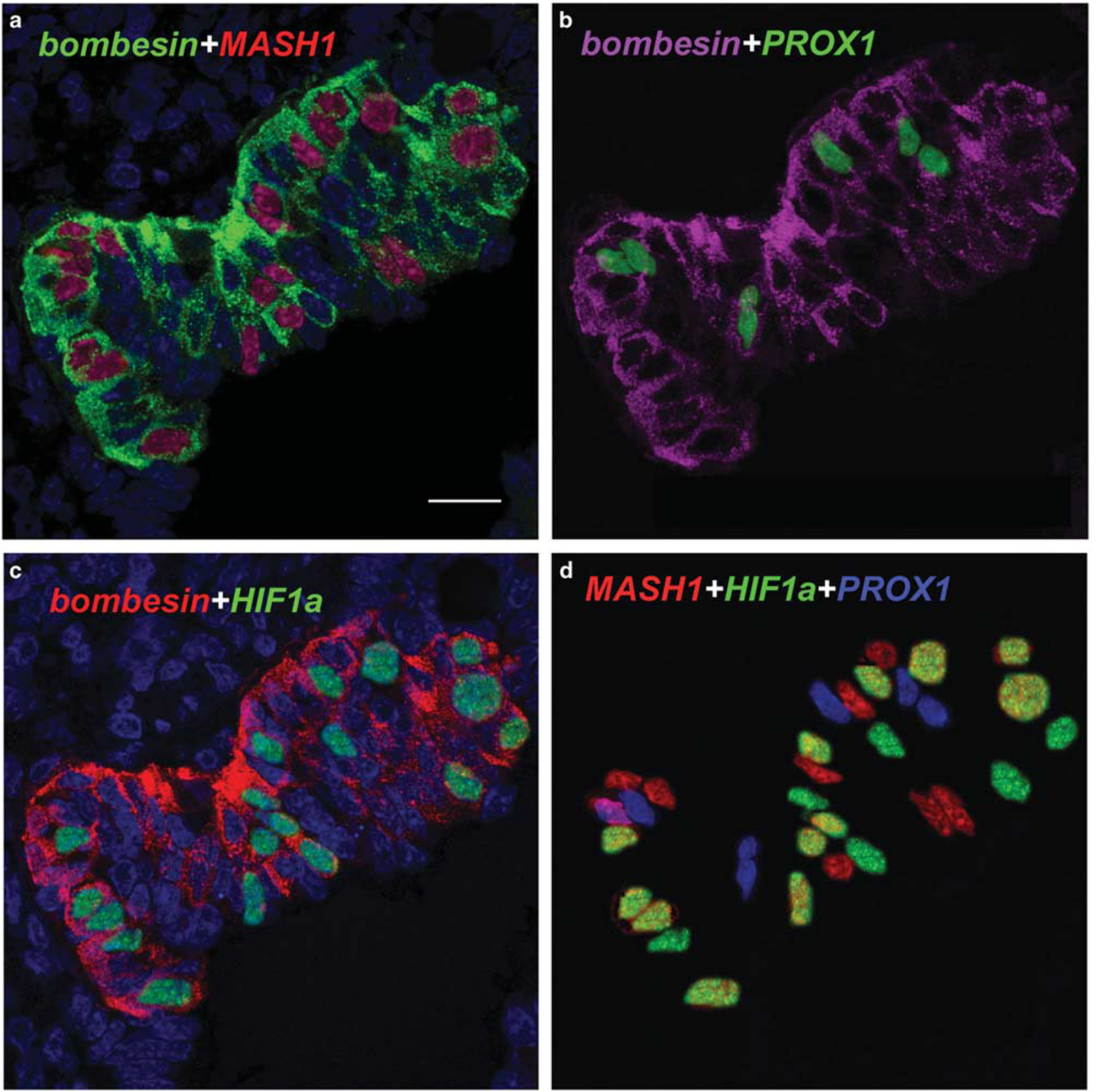

Figure 7 Tripple immunostaining of NEB in lung of 3-month-old infant with BPD using the same protocol as in Figure 6. (a) Immunostaining for bombesin (Texas red) and Mash1 (FITC, green) in hyperplastic NEB with up to 50 nuclei. Positive immunolabeling for Mash1 is present in 22 NEB cell nuclei $(<50 \%)$. (b) The same NEB after stripping and reprobing for bombesin (rhodamine, red) and Prox1 (FITC, green) with nuclear counterstain (Red Dot2 $687 \mathrm{~nm}$ in pseudo blue). Only occasional NEB cell nuclei express Prox1 ( 10\%). (c) Immunolabeling of same NEB as in a for HIF1 $\alpha$ showing numerous positive nuclei similar to Mash1 expression. (d) Merged image of immunostaining for three epitopes (Mash1, Prox1, and HIF1 $\alpha$ ) in the same NEB showing co-expression of Mash1 and HIF1 $\alpha$ in some nuclei with some nuclei exhibiting single epitope only. Scale bar $=10 \mu \mathrm{m}$.

CGRP in rodents. Although the precise function of NEBs neuroepithelial bodies is presently unknown, the predominant hypothesis suggests a role in respiratory control, particularly during the perinatal period when these cells are most prominent. $^{9,20}$ Whereas responses of NEB cells to acute hypoxia are mediated by ion channels, chronic sustained hypoxia involves the general cellular
$\mathrm{O}_{2}$ sensors, prolyl-hydroxylases, with downstream effects on expression of HIFs that in turn regulate expression of a large number of genes required for adaptation to a hypoxic environment. ${ }^{21}$ Previous studies identified hyperplasia of solitary PNEC and PNEC clusters, NEBs, in a number of pediatric lung disorders including chronic lung disease of prematurity, BPD PNEC and NEBs, which is associated 
with high morbidity and mortality. ${ }^{9}$ In addition, PNEC/NEBs are cells of origin of pulmonary neuroendocrine tumors such as carcinoid and small cell lung cancer. ${ }^{17}$ However, the mechanisms and pathobiology of PNEC/NEB cells proliferation and hyperplasia are poorly defined. In normal human lung and in cases of BPD, our MIH method was useful to co localize in the same cells, nuclear expression of neurogenic genes Mash1 and Prox1, required for PNEC/NEB cell differentiation, and/or a cell proliferation marker Ki67 together with cytoplasmic markers bombesin and SV2. Furthermore, we also demonstrate colocalization of neurogenic gene Mash1 with HIF1 $\alpha$. Our preliminary findings show that hyperplasia of NEB cells in lungs of patients with BPD is mediated predominantly by differentiation from precursor cells via Mash1 and Prox1 expression with a minor component of cell proliferation as demonstrated by sparse Ki67 labeling of NEB cells, compared with adjacent airway epithelium. Co-expression of Mash1 and HIF1 $\alpha$ suggests modulation by chronic hypoxia, known to be important in the pathobiology of BPD. ${ }^{22}$ The critical role of chronic hypoxia in PNEC/NEB cell hyperplasia is supported by our findings in PHD1deficient mice. The loss of PHD1 activity leads to continuous upregulation of HIF1 $\alpha$, mimicking chronic hypoxia exposure. PHD1 null mice showed two to threefold increase in NEB cell number and a similar expression pattern of neurogenic and cell proliferation markers as observed in infants with BPD suggesting that these mice could be a suitable animal model. ${ }^{23}$

Our reprobing method on FFPE sections is based on the same principles as conventional western blot reprobing, widely used in protein-based research. Here we show that previously immunostained FFPE tissue sections can be re probed after stripping the initial epitope with low $\mathrm{pH}$ and highly diluted SDS-containing solution. Remarkably, following this treatment the remaining epitopes retain their integrity and immunoreactivity allowing immunostaining with different epitope antibodies. This is particularly useful for colocalization of multiple cellular targets in the same cell/section and in cases when sample size and volume is limited.

We further suggest that these methods have a wide range of applications and are suitable for studies of a variety of cells and tissues including diagnostic pathology samples. The present report is a proof-ofprinciple and feasibility study demonstrating some examples of co-expression of multiple epitopes including plasma membrane, cytoplasmic, and nuclear localization in the same cells with high contrast and resolution. These methods are superior to currently widely used monochromatic, single epitope immunoperoxidase method. ${ }^{1}$ As we are introducing new methods to be used in diagnostic pathology, it is appropriate to discuss some practical issues such as the cost, technical expertize, the time required and the test validation. One of the limitations of our MIF method is the cost and availability of confocal microscope. However, this should not be a problem in Academic/University based Pathology laboratories (as in our case) where confocal microscopy is usually available as part of shared microscopy core facility. The cost of antibodies and various reagents is comparable to conventional immunohistochemistry. Future studies are required to carry out initial testing, validation, and applicability of proposed methods including the technical and laboratory-management aspects.

We believe that wide adoption of these methods to both pathobiology research and diagnostic pathology will lead to new discoveries as well as enhance diagnostic histopathology in a wide range of disease conditions including neoplasia, immunopathology, endocrine, and neuropathology etc. For example, the colocalization of oncogenes and specific cell surface receptors and/or nuclear epitopes in the same cells may be particularly useful for targeted antitumor therapies. ${ }^{24}$ Furthermore, as these methods produce digitized images, they are amenable to quantitative assessment of epitope expression. It is hoped that the adoption and application of our methods for the identification and colocalization of multiple epitopes in the same cells/tissues using FFPE sections will enhance diagnostic pathology practice and improve the accuracy of histopathology diagnosis, especially in cases where treatment decisions are based on detection of specific epitope expression required for individualized medical therapies. This is particularly relevant in the new era of 'Precision Medicine' initiative recently summarized by Drs Francis Collins and Harold Varmus. ${ }^{25}$ It is proposed that 'the initiative will encourage and support the next generation of scientists to develop creative new approaches for detecting, measuring, and analyzing a wide range of biomedical information-including molecular, genomic, cellular, behavioral, physiological, and environmental parameters'.

\section{Acknowledgments}

We thank Bernald Castro and Famida Spatare of the Division of Pathology, the Hospital for Sick Children for technical assistance; Dr Abdul Elkadri and Karoline Fiedler from the Division of Gastroenterology, Hepatology and Nutrition, the Hospital for Sick Children, and Dr Maureen O`Sullivan and Dr Seamus Hussey from Our Ladies Hospital Crumlin, Dublin, Ireland for providing patient material. Supported by CIHR grant (MOP-15270) to EC and HY, and by a RESTRACOMP stipend to CT from the Research Institute, The Hospital for Sick Children, Toronto, Canada.

\section{Disclosure/conflict of interest}

The authors declare no conflict of interest. 


\section{References}

1 Taylor CR. Immunohistochemistry in surgical pathology: principles and practice. In: Day CE (eds). Histopathology, Methods and protocols, Methods in Molecular Biology, 1180 Springer Protocols. Humana Press: Springer, NY, pp 81-109, 2014.

2 Nasr SH, Galgano SJ, Markovitz GS et al. Immunofluorescence on pronase-digested paraffin sections: a valuable salvage technique for renal biopsies. Kidney Int 2006;70:2148-2151.

3 Messias NC, Walker PD, Larsen CP. Paraffin immunofluorescence in renal pathology laboratory: more than salvage technique. Mod Pathol 2015;28:854-860.

4 Paik S, Kim C, Song Y et al. Technology insight: application of molecular techniques to formalin-fixed paraffin-embedded tissues from breast cancer. Nat Clin Pract Oncol 2005;2:246-254.

5 Spector DL, Goldman RD. Basic Methods in Microscopy Protocols and Concepts from Cells: a Laboratory Manual. Cold Spring Harbor Laboratory Press: Cold Spring Harbor, New York, NY, USA, 2006.

6 Müller T, Hess MW, Schiefermeier N, Pfaller K, Ebner HL et al. MYO5B mutations cause microvillus inclusion disease and disrupt epithelial cell polarity. Nat Genet 2008;40:1163-1165.

7 Sivagnanam M, Mueller JL, Lee $\mathrm{H}$ et al. Identification of EpCAM as the gene for congenital tufting enteropathy. Gastroenterology 2008;135:429-437.

8 Gillan JE, Cutz E. Abnormal pulmonary bombesin immunoreactive cells in Wilson-Mikity syndrome (pulmonary dysmaturity) and bronchopulmonary dysplasia. Pediatr Pathol 1993;13:165-180.

9 Cutz E, Yeger H, Pan J. Pulmonary neuroendocrine cell system in pediatric lung disease-recent advances. Pediatr Dev Pathol 2007;10:419-435.

10 Thoeni CE, Vogel GF, Tancevski I et al. Microvillus inclusion disease: loss of myosin vb disrupts intracellular traffic and cell polarity. Traffic 2014;15:22-42.

11 Thoeni C, AmirA, Guo C et al. Novel non sense mutation in the EpCAM gene in a patient with congenital tufting enteropathy. J Pediatr Gastroenerol Nutr 2014;58:18-21.

12 Canani RB, Castaldo G, Bacchetta R et al. Congenital diarrhoeal disorders: advances in this evolving web of inherited enteropathies. Nat Rev Gastroenterol Hepatol 2015;12:293-302.
13 Pan J, Yeger H, Cutz E. Innervation of pulmonary neuroendocrine cells and neuroepithelial bodies in developing rabbit lung. J Histochem Cytochem 2004;52: 379-389.

14 Lei Z, Maeda T, Tamura A et al. EpCAM contributes to formation of functional tight junction in the intestinal epithelium by recruiting claudin proteins. Dev Biol 2012;371:136-145.

15 Keitel V, Burdelski M, Warskulat U et al. Expression and localization of hepatobiliary transport proteins in progressive familial intrahepatic cholestasis. Hepatology 2005;41:1160-1172.

16 Mc Govern S, Pan J, Oliver G et al. The role of hypoxia and neurogenic genes (Mash-1 and Prox-1) in the developmental programming and maturation of pulmonary neuroendocrine cels in fetal mouse lung. Lab Invest 2010;90:180-195.

17 Miki M, Ball DW, Linnoila RI. Insights into achetescute homolog -1 gene (hASH1)in normal and neoplastic human lung. Lung Cancer 2012;75:58-65.

18 Ruemmele FM, Müller T, Schiefermeier $\mathrm{N}$ et al. Loss-of-function of MYO5B is the main cause of microvillus inclusion disease: 15 novel mutations and a CaCo-2 RNAi cell model. Hum Mutat 2010;31: 544-551.

19 Wiegerinck CL, Janecke AR, Schneeberger K et al. Loss of syntaxin 3 causes variant microvillus inclusion disease. Gastroenterology 2014;147:65-68.e10.

20 Cutz E, Pan J, Yeger $\mathrm{H}$ et al. Recent advances and controversies on the role of pulmonary neuroepithelial bodies as airway sensors. Sem Cell Dev Biol 2012;24: 40-50.

21 Kaelin WG, Ratcliffe PJ. Oxygen sensing in metazoans: the central role of the HIF hydroxylase pathway. Mol Cell 2008;30:393-402.

22 Baraldi E, Filippone M. Chronic lung disease after premature birth. New Engl J Med 2007;357: 1946-1955.

23 Pan J, Yeger H, Radcliffe $\mathrm{P}$ et al. Hyperplasia of pulmonary neuroepithelial bodies in lungs of prolylhydroxylase-1 deficient mice. Adv Exp Med Biol 2012;758:149-155.

24 Duffy MJ, O’Donovan N, Crown J. Use of molecular markers for predicting therapy response in cancer patients. Cancer Treat Rev 2011;37:151-159.

25 Collins FS, Varmus H. A new initiative on precision medicine. New Engl J Med 2015;372:793-795. 